\title{
A Web-based Benchmarking System for Health Research Scientists, Case Study - Saudi Arabia
}

\author{
Tahani Daghistani \\ MS in Health Informatics, King Saud Bin Abdulaziz \\ University for Health Science \\ King Abdullah International Medical Research \\ Center (KAIMRC) \\ Ministry of National Guard Health Affairs (MNGHA) \\ Riyadh, Saudi Arabia
}

\author{
Hanan Althagafi \\ Ministry of National Guard Health Affairs (MNGHA) \\ P.O Box 54178 \\ Zip Code 11541 \\ Riyadh, Saudi Arabia
}

\begin{abstract}
Objectives: The primary objective is to explore the Research Award System (RAS) experience at King Abdullah International Medical Research Center (KAIMRC) in Saudi Arabia. Methods: A case study method was used to describe a real experience of a research award system (RAS) with multiple data sources collection. Results: RAS is one of the tools used by KAIMRC for attracting and retaining research scientists and improve the overall performance, in line with its vision and strategies. RAS identifies, validates and honor research scientists based on quantitative measures. It covers three groups of research scientists and calculate the final score(FS) for a total of ten (10) criteria and based on the assigned criterion/sub-criterion weight. The Journals Impact Factor (JIF) that required for the calculations, can be imported from outsourcing databases like International Scientific Indexing (ISI) or Scopus. Also, provides each applicant with an estimated final score (FS) instantly. Conclusion: The current case study highlights a unique experience represented in an automated research award system (RAS). RAS automates the process of selecting winners using quantitative criteria and benchmarking approach. Such a system can be utilized at broader levels, to support research institutions or $R \& D$ organizations in self-assessment on the level of their belonging research scientists and to develop a large pool of research scientists data required for research programs and initiatives.
\end{abstract}

\section{Keywords}

Research Award, Web-Based, Benchmarking, Performance, Scoring System, Self-Assessment

\section{INTRODUCTION}

Healthcare and research services organizations are leading their research capacity in a dynamic and competitive environment. Building research capacity has become a significant aspect in health arena at multiple levels, due to its impact on improving population health and wealth that are required for the sustainable development [1]. Several studies have shown that financial depth, protection of intellectual property rights, government capacity to mobilize resources, and the quality of research institutions appear as the main reasons for high investing in research [2].

In Saudi Arabia where the financial and government support for research was considered high in the MENA region during the last 10 years, it is valuable for the research institutions to focus their efforts on improving the research productivity in terms of quality and quantity. In the view of research capacity, research institutions in Saudi Arabia should initiate programs to emerge research recognition, research learning and research return on investment. Taking into account, factors that may impact potential and expert research scientists, to stay encouraged and preserve a positive attitude. From this standpoint, Research competitions can be organized as one way to achieve this goal. A critical question that can be posed is how do the performance of research productivity measured? As an example, the high number of publications such as articles, conferences, books etc. , is a recognized indication of a research productivity and evolving knowledge successfully $[3,4]$. However, the practice of research evaluation can be defined as an activity in which certain aspects of the quality of research practice are investigated. Since there is no correct answer for the previous question, regarding matrices that use to measure research output or research performance [5], tangible or quantitative measures are needed to evaluate a wide majority of researchers systematically and scientifically [6]. Quantitative measures are different than peer- review in which peer review information provided by multiple experts by multiple qualitative evaluation criteria. Although, peer review research evaluation is convenient for educational purposes [7], yet it still popular and used in many journals and research projects evaluation panels [8].

Accordingly, Benchmarking method can be used on quantitative measures for greater efficiency in research or research scientists' evaluation. Taking into consideration, benchmarking approach can be meaningless if invalid criteria were used. It has been known that performance criteria presented outside a benchmarking method have no power to initiate revolution for improvement [9]. Moreover, the importance and impact of research within the certain context of the organization are enabling to feed such measurements to continuous improvement cycle.

Presently, there is an automated system for measuring and benchmarking the performance of research productivity in Saudi Arabia. This system was developed and launchedbefore a couple of years- by a well-known local research institution to measure many aspects of research outcomes with the aim of covering existing and potential research scientists in the country.

It is the intention of this paper, to provide a convenient mechanism by which any organization can assess their research productivity performance and compare it against other organizations [9]. The general aim of this case study is to discover the use of performance criteria to measure and benchmark research productivity performance. The primary objective is to explore Research Award System experience at King Abdullah International Medical Research Center (KAIMRC) in Saudi Arabia. 


\section{STUDY DESIGN}

\subsection{Study Setting}

King Abdullah International Medical Research Center (KAIMRC) is a research institution Operates under the umbrella of the King Saud Bin Abdulaziz University for Health Sciences (KSAU_HS) and Ministry of National Guard Health Affairs (MNGHA). KAIMRC has multiple branches located in Riyadh, Al Ahsa, Dammam, Jeddah, and AlMadinah. It aims to be a leading institution in biomedical and clinical research. Currently, KAIMRC focus on a few selected disease areas (Cancer, Diabetes, Cardiovascular, Infectious, Neurological) which align with national strategic priorities. In addition to other research projects such as Saudi Biobank, Cord Blood Bank, and the Research Trauma Project [10].

\subsection{Technology}

Research Award System is a web-based benchmarking system, developed by in-house IT developers based on Microsoft CRM. It is an application that measure and benchmark research scientists based on ten (10) criteria. The purpose of this system is to streamline the submission and automate the process of selecting the winner. It is a scoring system that ranks research scientist based on ten (10) criteria benchmark calculation. Benchmark is probably the most widely known approaches wherein the performance of a given research scientists is compared against the highest research scientist's performance [9].The users can access the system by using their identification badge and should have an affiliation to KAIMRC, KSAU-HS or MNGHA

\subsection{Methodology}

A case study method was used for the current study. A case study from KAIMRC that describes a real experience in which the research award system has been implemented. As well as detailed information from different sources has been put forward for discussion. The data collection sources used for the current case study are system documents, interviews with expertise from research business and technical side, users in addition to reviewing a variety of related articles.

\section{RESULTS}

\subsection{Experience from Saudi Arabia}

Research award is one of the strategies used by KAIMRC for attracting and retaining research scientists to improve the overall performance, in line with the vision and strategies. As a result, KAIMRC seeks to implement automated Research Award System (RAS) that identifies, validates and honor research scientists based on the KAIMRC policies and regulations. Similar systems usually used as a mechanism that enables organizations to reach their target [1]. The purpose of the RAS is to provide an automated and systematic way to measure and benchmark research productivity belong research scientists. Technically, RAS has three (3) types of users:

- The administrator who has full access to the system components and full permission.

- The reviewer who verifying data entered by the applicants.
- The applicant who has limited access to enter the required data, edit the data before the submitting the application.

Initially, the research scientist should meet the eligibility requirements, which have been identified by a special Research Award Committee, and before the self-nomination by applying to RAS. Figure 1 shows the road map (1 to 16 steps) of RAS way of working. The data submitted in the RAS is accumulative data, the applicant needs to update his/her profile only, as the competition conducted annually. The RAS provides a limited ability to import information required for calculations such as Journal Impact Factor (JIF) from outsourcing databases like International Scientific Indexing (ISI) or Scopus. The RAS covers three groups of research scientists:

- $\quad$ Senior Group: where the research scientist (applicant) should have an extensive level of expertise in the scientific research by at least fifteen (15) years' experience.

- Experienced Group: where the research scientist (applicant) should have a good level of expertise in the scientific research, less than fifteen (15) years of experience and the age of the scientist is more than 35 years old.

- Beginner Group: where the research scientist (applicant) should is less than 35 years old and have a record of research productivity.

The RAS calculated the total benchmark number for a total of ten (10) criteria and based on the assigned criterion weight (Figure 2). The criterion weight (criterion weight) assigned (1) will be used to calculate the applicant's Raw Score (RS) for each criterion. After the submission of all applications, the highest attained score for each criterion (Criterion Benchmark Score (CBS)) calculated in order to attain applicant's Benchmarking Score (ABS for an applicant for a selected criterion) using equation-1:

Applicant's benchmarking Score $=$

(Raw Score / Benchmark Score) * Maximum Benchmark Score

$$
\mathrm{ABS}=(\mathrm{RS} \div \mathrm{CBS}) * \text { Criterion weight }(1)
$$

Then, the system calculates the Final Score (FS) which is the accumulated total score obtained for an applicant by using equation -2 :

$$
\mathrm{FS}=\Sigma(\mathrm{ABS})
$$

The RAS provides each applicant, an estimated final score (FS), once completed his/her submission. However, the estimated final score is not fixed, as ongoing change are reflected in the benchmark score, due to the other applicant's' submission. By the end of the nomination period, the calculation of the scores will be final and the system will be locked for any submission or editing. 


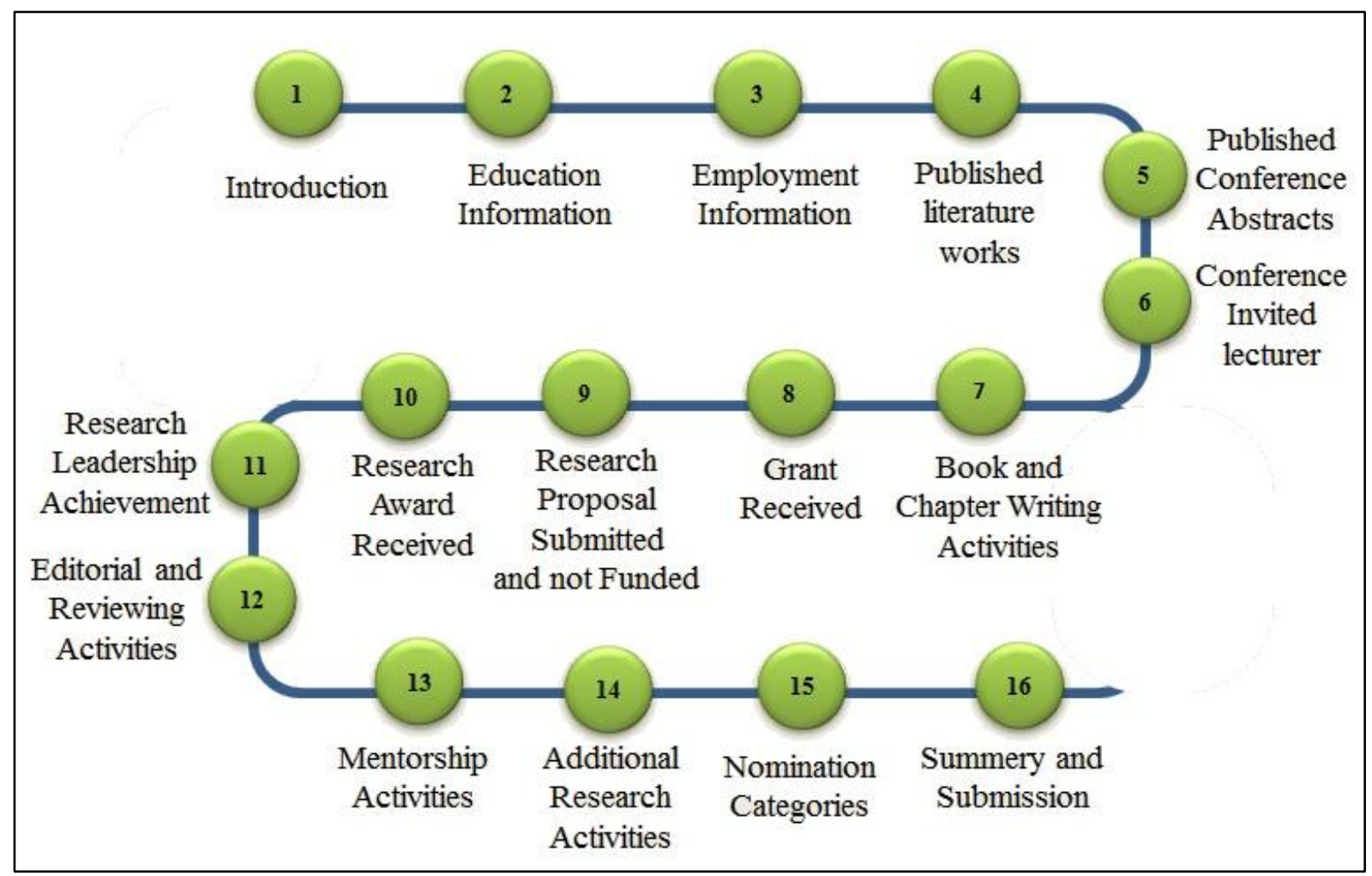

Fig. 1: The Road Map of Research Award System (RAS) Steps

\subsubsection{Example\#1: Showing the steps of calculating final score (FS) of an applicant in RAS -Published Literature Criterion}

- Calculate Raw Score (RS):

Published literature Criterion will be calculated based on the total of citation scores. The citation score value is based on the Journal Impact Factor (JIF) updated value (source: ISI). Moreover, If the applicant in his publication, was the first and/or corresponding Author (value $=1$ ), or other (value $=$ $0.25)$.

Citation score for each publication= Journal Impact Factor (JIF) * Author Rank

IF ("1st author/corresponding author" then (1* Impact Factor)

Else ("Subsequent Author" then (0.25 *Impact Factor)

Citation Score for a given publication: $3.6 * 0.25=0.9$; when JIF is 3.6 and the rank of the author is other

The Raw Score of published literature works criterion= total of the citation scores.

- Identify Criterion Benchmark Score (CBS):

The criterion benchmark score will be identified from the highest score obtained for this criterion of all applicants. For example, in published literature works criterion, the raw scores of all applicants were between 85-125, so the highest score is 125 which considered as the benchmark score (CBS) for this criterion

- Calculate Applicant Benchmarking Score: Applicant Benchmarking Score $(\mathrm{ABS})=(\mathrm{RS} \div \mathrm{CBS}) *$ Criterion Weight
If the Criterion Weight for Published Literature is $30 \%$, and the lowest registered RS is (90) for applicant-A and the highest registered RS is (130) for applicant-B

Then

ABS For the applicant-A is equal to $(90 \div 130) * 30 \%=0.21$

ABS for applicant-B is equal to $(130 \div 130) * 30 \%=0.3$

- Calculate Final Score:

The final score of an applicant will be calculated by aggregate all the calculated ABS values.

$$
\text { Applicant Final Score }=\Sigma(\mathrm{ABS})
$$

\subsubsection{Example\#2: Showing the steps of} calculating final score (FS) of an applicant in RAS - Editorial Productivity Criterion

- Calculate Raw Score (RS):

Editorial Productivity for an applicant will be calculated based on the publishing house classification (international or national (50\% of International values)), Also, if the applicant was a Chairman/ Leader/ Head (value = 3), an Assessor (value $=2$ ), or other (value $=1$ ).

IF ("International"

Then

IF (Type="Chief Editorial Board"

Then 3

Else

IF (Type ="Editorial Board Member"

Then 2 
Else 1)),

Else

IF $($ Type $="$ Chief Editorial Board"

Then $(3 * 0.5)$

Else

IF (Type ="Editorial Board Member"

Then $(2 * 0.5)$

Else $(1 * 0.5)))$ )

The Raw Score of a given Editorial Activity: $3 * 0.5=1.5$; when the publishing house is national, and the role of the applicant is Chief Editorial Board.

The Raw Score of editorial productivity criterion= total of the editorial scores.

- Identify Criterion Benchmark Score (CBS):

The criterion benchmark score will be identified from the highest score obtained for this criterion of all applicants. For example, in editorial productivity criterion, the raw scores of all applicants were between 16- 20, so the highest score is 20 which considered as the benchmark score (CBS) for this criterion.

- Calculate Applicant Benchmarking Score:

Applicant Benchmarking Score $(\mathrm{ABS})=(\mathrm{RS} \div \mathrm{CBS}) *$ Criterion Weight

If the Criterion Weight for Editorial Productivity is $15 \%$, and the lowest registered RS is (16) for applicant-A and the highest registered RS is (20) for applicant-B

Then

ABS For the applicant-A is equal to $(16 \div 20) * 15 \%=0.12$

ABS for applicant-B is equal to $(20 \div 20) * 15 \%=0.15$

- Calculate Final Score:

The final score of an applicant will be calculated by aggregate all the calculated ABS values.

Applicant Final Score $=\Sigma($ ABS $)$

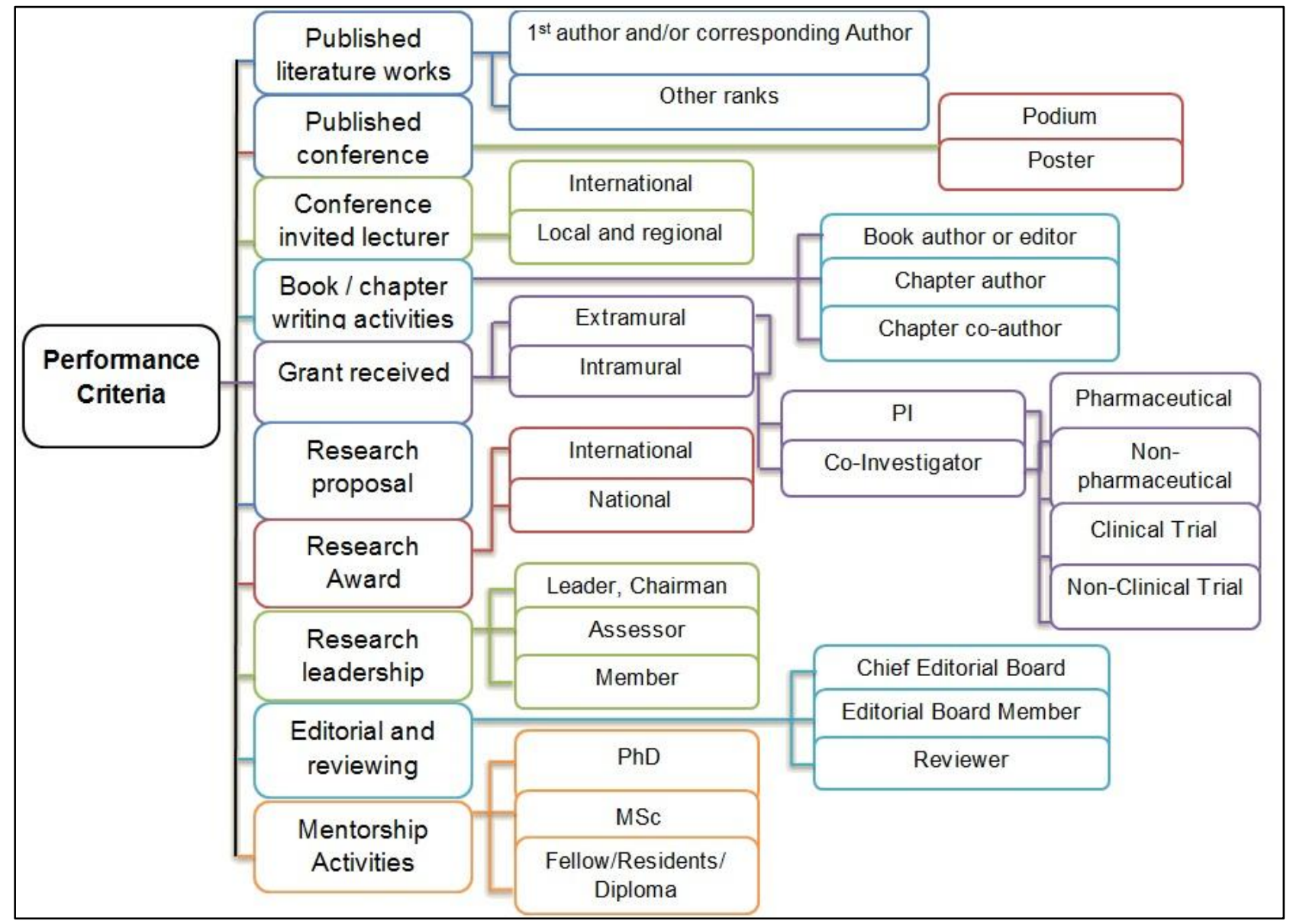

Fig. 2: Criteria of Research Award System (RAS)

\section{DISCUSSION}

It's known that rewarding research scientists on their research productivity efforts are relevant to make the research field, more attractive and empowering the research environment for the aim of research retention and growth. It provides an opportunity for success, advancement of researcher scientists and influences research institutions to attract more research scientists and funds [11, 4]. Specifically, a financial reward which can have a strong impact on the research behavior in conducting multidisciplinary research with high quality [4]. Several studies conducted in many aspects of the researchoriented award which covered topics relevant to performance, productivity, motivation, satisfaction and quality of work [12, 13]. Overall the studies showed that reward has a positive 
effect. But there are mixed conclusions when it comes to different groups of the award and their effect [1]. A study conducted by Antoine Danchin [3] concluded that institutions should play an active role in helping researchers in the early years of their career to make research area, attractive for potential research scientists or who have preliminary records of research publications, which exists in the current research Award system as the beginner researcher group.

The current case study demonstrated a well-recognized research award system that designed to evaluate the research efforts of research scientists in the healthcare sector. The same system could be used to measure and benchmark the performance of research scientists within any concerned organization from different sectors. Additionally, this might be more comprehensive to cover the international level if further studies conducted on the validation of the research award criteria. However, the lack of consistency in methods used among organizations is one of the main difficulties with benchmarking $[9,14]$. In the existing research award system, it focuses mainly on the quantity of research over a timeframe and based on rigorous criteria [12]. There are ten (10) criteria for the three (3) research award groups. The publication literature criterion, for example, has counted the number of publications for a given research scientists [9], taking into consideration the Journal Impact factor of each publication as an indication of the quality, not a quantity $[9,15,16,17]$, yet it provides no indication of the impact of the publications. It would be more comprehensive if the system can utilize citation analysis methods, such as a number of citations, selfcitations, or a reason for citing (support findings, opposes findings). As per previous studies, citation analysis is a more reliable tool to investigate the impact of publications rather than the only count of publications [18]. The existing research award system can easily import the citation index such as Hirsch-index (h-Index) with minor technical customization to the system and the hosting server. A subscription to Scopus or the Web of Knowledge databases, to import the h-index calculated attributes may require. Or importing the citation index directly from online sources of citation data including Google Scholar, Google Books, Google Blogs, which are sufficient to be useful for the impact assessment of research [19]. Moreover, the h-index reflects both the number of publications and the number of citations per publication, but it's recommended to take into account that citation behavior, in general, is affected by field-dependent factors, which may invalidate comparisons not only across disciplines but even within different fields of research of one discipline. As a result, the existing research award system has some limitations regarding the citation analysis which considered in another similar research award such as Research Productivity Award administered by Pakistan Council for Science and Technology[20]. The variation in the impact factor values between medical journals and non-medical journals was observed, the academic- oriented applicants should double their publication numbers in order to compete with medical applicants. Thus, it is recommended to check the homogeneity of the possible applicants before the announcement or apply RAS in separate tracks.

Additionally, there is no evidence that conference publication (podium or posters) is a valid indication of the expertise of research scientists. In fact, conference publication sometimes rigorous, prestigious and requires evaluation from knowledgeable peers, which may be suitable for certain science disciplinary such as a computer, engineering [21], or applied medical research. Also might preferable for beginner expertise group which may show how much they active in their field. Indeed, these criteria need more validation and reliability tests and to more investigation on its correlation with the final score or the final benchmark score.

Other criteria such as patent, grant, research awards, book/chapters publications and research productivity (mentorship for Ph.D. dissertations) are logically indicators to measure and benchmark the research scientists; if the face validity considered. Yet, the book online citation can be used as an attribute for Book/chapter calculation. But further validity investigation is required too. With a note that, some of these previous criteria such as patent and grant might be suitable more for highly expert research scientists, and not properly applied for beginner research scientists group.

In the end, the Saudi Arabia government works to develop the national youth capacity in the fields of innovation and applied science. Accordingly, a customization to the existing research award system is recommended to focus on the specific scope of applied medical research and other fields such as business intelligent research. The existing research award system can be utilized by the higher authority of health in SA (MOH) as a tool to evaluate and lead the scientific research for the most needed fields. Also, it can be used as a tool for scientists promotion eligibility, which reflects a reliable competencies index. In fact, the existing research award system needs further customization and investigation on its validity, reliability and its impact on the institutional performance and quality of care since it was applied for several years in MNGHA

\section{CONCLUSION}

The current case study highlights a unique experience, at KAIMRC in Saudi Arabia. The automated research award system (RAS) automates the process of scoring \& benchmarking research scientists based on pre-defined quantitative criteria related to quality of research and publications. Criteria standards built on evidence- based literature review and local expert panel input. literature review was critical to identify the most validated indicators such as Journal impact factor, citation score, and the total number of publications. In addition to the local expert panel, which has two major tasks, first to quantify the qualitative research evaluation criteria such as authorship ranks and criterion rank. The second task is to provide a kind of peer- review information when needed. And therefore, the real time benchmarking will be applied automatically.

The mentioned system (RAS) went through several reforms during the past couple of years to ensure its relevance and support to the KAIMRC strategy. As the award gained popularity, locally, the diversity of registrants increased to include non-medical specialties such as health informatics, health management and bio- medical engineering. Consequently, large pool of local research scientists data in (RAS) emphasizes the need to exploring the reliability and validity, which may later be a subject for further study. Besides, it can be used as a reference for more scientific research programs and initiatives [14].

Indeed, (RAS) system can be used on a broader level. At the national \& regional levels, it can be used to benchmarking research institutions in diverse sectors. As well supporting the GCC governments in their strategies of building R\&D infrastructure. RAS enhances the value and growth of the scientific researchers, by utilizing (RAS) as a self-assessment tool. RAS can be useful in the area of human resources management, capacity development, hiring, and promoting processes. 


\section{REFERENCES}

[1] Njanja LW, Maina RN, Kibet LK, Njagi K. Effect of reward on employee performance: A case of Kenya Power and Lighting Company Ltd., Nakuru, Kenya. International Journal of Business and Management. 2013 Oct 15;8(21):41.

[2] Lederman D, Maloney WF. R\&D and development. Available at SSRN: https://ssrn.com/abstract $=402480$

[3] Danchin A. Motivated research. EMBO reports. $2010 \mathrm{Jul}$ $1 ; 11(7): 488-$.

[4] Zhang X. Factors that Motivate Academic Staff to Conduct Research and Influence Research Productivity in Chinese Project 211 Universities (Doctoral dissertation, University of Canberra).

[5] Wootton R. A simple, generalizable method for measuring individual research productivity and its use in the long-term analysis of departmental performance, including between-country comparisons. Health research policy and systems. 2013 Jan 14;11(1):2.

[6] Bornmann L, Marx W. How to evaluate individual researchers working in the natural and life sciences meaningfully? A proposal of methods based on percentiles of citations. Scientometrics. 2014 Jan $1 ; 98(1): 487-509$.

[7] Dopico E. Peer Review as Teaching Method. Educ Elearn. 2016;1(001). Available at: http://crescopublications.org/eeoa/EEOA-1-001.pdf. [Accessed 6 May 2017].

[8] Zhu WD, Liu F, Chen YW, Yang JB, Xu DL, Wang DP. Research project evaluation and selection: an evidential reasoning rule-based method for aggregating peer review information with reliabilities. Scientometrics. 2015 Dec 1;105(3):1469-90

[9] Meek VL, van der Lee JJ. Performance indicators for assessing and benchmarking research capacities in universities. Background Paper prepared for the Global University Network for Innovation-Asia and the Pacific, UNESCO-Bangkok. 2005 May.

[10] Medical Research Center (KAIMRC) [Internet]. Ksauhs.edu.sa. 2017 [cited 6 May 2017]. Available from: http://www.ksau-hs.edu.sa/English/Research/Pages/ KAIMRC.aspx

[11] Mason S, Marchetti CE, Bailey MB, Baum S, Valentine M. Faculty Awards at a Large Private Institution: An Indicator of Evolving University Values?. InProc. 2014
American Society for Engineering Education Annual Conference \& Exposition 2014

[12] Bonaparte I, Abbey A, Okoro E. Challenges facing beginning Faculty in the 21st Century Higher Education: Evaluating Research Productivity, Teaching Effectiveness, And Service. International Journal of Academic Research in Progressive Education and Development. 2014;4(1):143-62.

[13] Neckermann S, Cueni R, Frey BS. Awards at work. Labour Economics. 2014 Dec 31;31:205-17.

[14] Cetindamar D, Kilitcioglu H. Measuring the competitiveness of a firm for an award system. Competitiveness Review: An International Business Journal. 2013 Jan 18;23(1):7-22.

[15] Jain AK. Impact factor: Measure of quality of research publication. Indian journal of orthopaedics. 2011 Jul;45(4):289.

[16] Khan KM, Hegde P. Is impact factor true evaluation for ranking quality measure?. DESIDOC Journal of Library \& Information Technology. 2009 May 1;29(3):55.

[17] Saha S, Saint S, Christakis DA. Impact factor: a valid measure of journal quality?. Journal of the Medical Library Association. 2003 Jan;91(1):42. Available at: http://vietsciences.free.fr/khaocuu/nguyenvantuan/Osteo porosis/IF.pdf. [Accessed 6 May 2017]

[18] King J. A review of bibliometric and other science indicators and their role in research evaluation. Journal of information science. 1987 Oct;13(5):261-76. Available http://citeseerx.ist.psu.edu/viewdoc/download?doi=10.1. 1.979.9771\&rep $=$ rep1\&type $=$ pdf $\quad$ Accessed 5 May 2017].

[19] Kousha K, Thelwall M, Rezaie S. Using the web for research evaluation: The Integrated Online Impact indicator. Journal of informetrics. 2010 Jan 31;4(1):12435 .

[20] Pakistan Council for Science and Technology [Internet]. Pcst.org.pk. 2017 [cited 6 May 2017]. Available from: http://www.pcst.org.pk/rpa.php

[21] Page A. Best Practices Memo Evaluating Computer Scientists and Engineers For Promotion and Tenure. Computing. 1999 Sep. Available at: http://archive2.cra.org/uploads/documents/resources/bpm emos/tenure_review.pdf. [Accessed 6 May 2017]. 